Document downloaded from:

http://hdl.handle.net/10251/180567

This paper must be cited as:

Vraka, A.; Osorio, D.; Bertomeu-González, V.; Ravelli, F.; Alcaraz, R.; Rieta, JJ. (2019).

Detection of Dominant Reentries in Multichannel Electrograms of Atrial Fibrillation. IEEE. 1-

4. https://doi.org/10.1109/EHB47216.2019.8969992

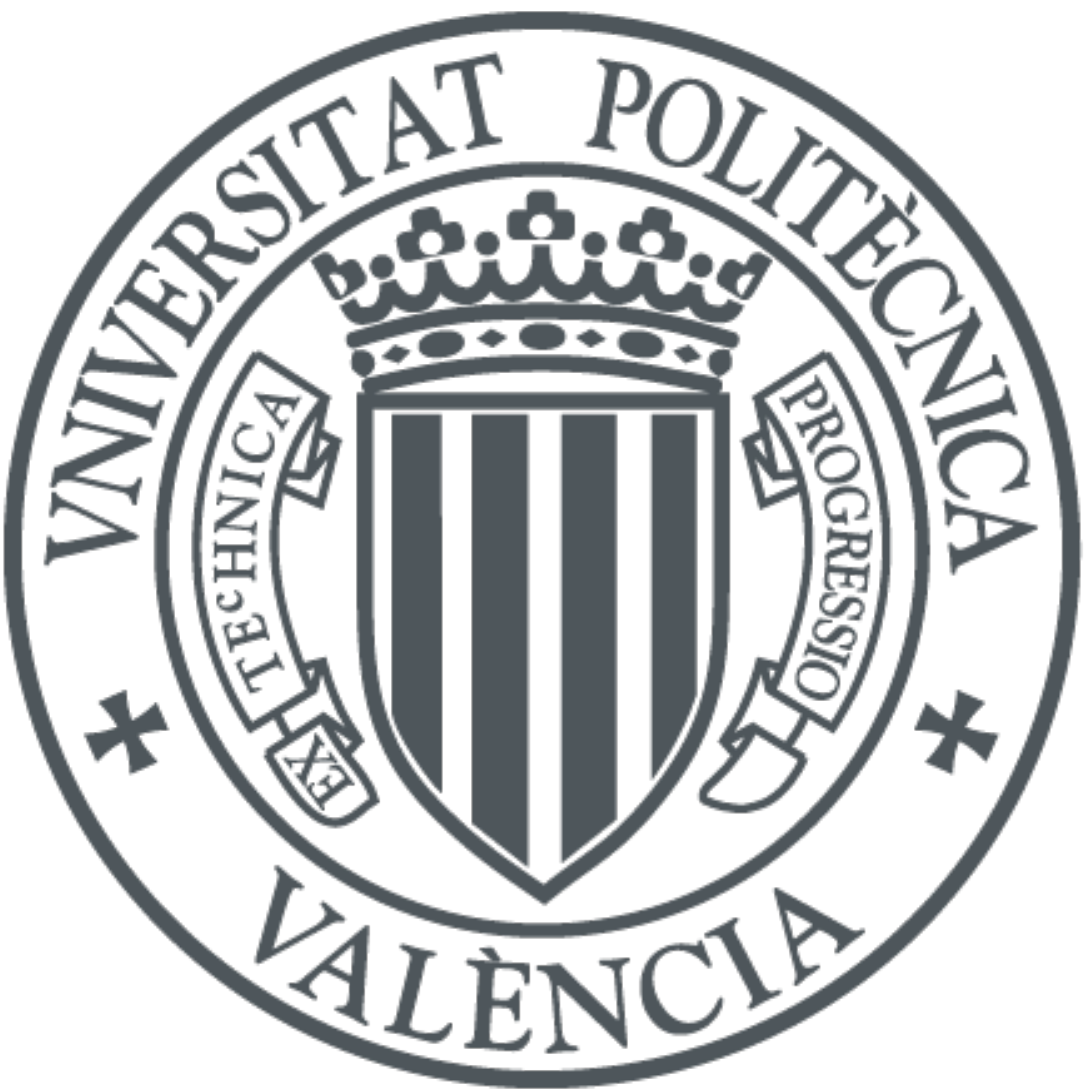

The final publication is available at

https://doi.org/10.1109/EHB47216.2019.8969992

Copyright IEEE

Additional Information 


\title{
Detection of Dominant Reentries in Multichannel Electrograms of Atrial Fibrillation
}

\author{
Aikaterini Vraka ${ }^{1}$, Diego Osorio ${ }^{1}$, Vicente Bertomeu-González ${ }^{2}$, Flavia Ravelli ${ }^{3}$, Raúl Alcaraz ${ }^{4}$, José J. Rieta ${ }^{1}$ \\ ${ }^{1}$ BioMIT.org, Electronic Engineering Dep., Universitat Politecnica de Valencia, Spain; \{aivra, dosorio, jjrieta\}@upv.es \\ 2 Cardiology Department, Hospital Universitario de San Juan, Alicante, Spain; vbertog@ gmail.com \\ ${ }^{3}$ Department of Physics, University of Trento, Italy; flavia.ravelli@unitn.it \\ ${ }^{4}$ Research Group in Electronic, Biomedical and Telecomm. Eng., Univ. of Castilla-La Mancha, Spain; raul.alcaraz@uclm.es
}

\begin{abstract}
Algorithms developed so far for the detection of local activation waves (LAWs) in electrograms (EGMs) of atrial fibrillation (AF) analyze each channel of the catheter separately, while dominant reentries of AF are captured by most of the catheter channels simultaneously. This encompasses the risk of losing or oversensing activations in the case of complex fractionated atrial electrograms (CFAEs). The proposed method analyzes multichannel recordings of AF from the least to the most fractionated. Activations are manually annotated and then EGMs are denoised, band-pass filtered by an alternative Botteron approach and thresholded. LAW detection is performed sequentially on all the channels by ascending fractionation order. Activations in the first EGM are found by an amplitude-based search, followed by a cycle length (CL) based search. Detection algorithm in the remaining signals is based on the previously detected LAWs, applying a varying search window according to the repetition rate of each activation. A CL-based search is also applied and activation time is computed from the barycenter of each LAW. The final step contains a secondary control that deletes all the low amplitude activations showing low repetition rate, so that any bias regarding the LAW detection of the first EGM is avoided. The method was compared with the equivalent sequential single-channel analysis, with the former showing better accuracy $(90.82 \%)$ and positive predictive value $(96.52 \%)$ than the single-channel approach $(89.65 \%$ and $92.70 \%$, respectively). Multichannel analysis performed a more selective analysis, ignoring some obvious but not repeated LAWs. Consequently, it can localize AF triggers and propagation phenomena more robustly than single channel method.
\end{abstract}

Keywords-Atrial fibrillation; catheter ablation; complex fractionated atrial electrograms; multichannel; local activation waves.

\section{INTRODUCTION}

Atrial fibrillation (AF) is the most common cardiac arrhythmia, with its prevalence expectancy marking the score of one out of four middle-aged adults in Europe and the US [1]. As AF is accompanied with high mortality risk [2] from the very first stages of the diagnosis [3], acting quickly with the suitable treatment is of paramount importance. However, as triggering and perpetuating activity differs from case to case, the choice of the adequate treatment should be done taking into consideration each patient's particularities, so that precise mapping of AF mechanisms is necessary.

AF related therapies focus in the first place on the maintenance of sinus rhythm and on the control of the atrial rate [4], while catheter ablation (CA) is currently the most popular therapy for sinus rhythm restoration [5]. During CA procedure, high-energy radiofrequency signals make scars in the heart tissue, isolating the areas that present arrhythmogenic or AF perpetuating properties. Real-time mapping of the atrial tissue should hence be performed with high precision and accuracy. This way the ablation catheter can be effectively navigated and clinicians gain a complete perspective of the AF substrate, so that unnecessary scars can be avoided.

Understanding the mechanisms that either generate or perpetuate the AF is a crucial step for the definition of its pathophysiologic substrate. Pulmonary veins (PVs) are thought to be the most common AF triggers [6] and therefore, pulmonary vein isolation (PVI), is up to now the most widespread AF treatment in paroxysmal AF patients.

As persistent $\mathrm{AF}$ is more complicated than paroxysmal, the recurrence rates of PVI in this stage are quite high. In persistent $\mathrm{AF}$ cases, $\mathrm{AF}$ activity spreads through other sites of the atrium and the coronary sinus (CS), favoring the transmission of ectopic signals [7]. Ablation of other areas that are active to AF perpetuation should hence be considered, in addition with the PVI [8].

Cardiac signals obtained through recording catheters are called electrograms (EGMs) and differ from electrocardiograms (ECGs), which record the signal from the surface of the body [9]. Sites with arrhythmogenic or AF perpetuating activity often produce EGMs with high fractionation [10]. Complex fractionated atrial electrograms (CFAEs) is a category of EGMs that have often been targeted as AF drivers [11]. CFAEs are highly fractionated atrial EGMs that exhibit multiple deflections from the isoelectric line and/or potentials with continuous electrical activity without an isoelectric line [11]. High complexity of these EGMs and the frequent lack of clear activations can make the detection of local activation 
waves (LAWs) a rather difficult task with ambiguing results that strongly affect the AF treatment.

The importance and complexity of LAW detection, especially regarding CFAEs, has lead to the development of different algorithmic approaches. Some of them use an adaptive threshold and base their analysis on waveform morphology similarity [12] while others focus only on the periodic activations, depending on the dominant frequency (DF) of the EGMs [13], with the risk of mishits in case of CFAEs, where DF varies significantly. Another technique with interesting results is cycle length (CL) based detection [14].

All these techniques analyze each channel separately, whereas the recording catheter is a multichannel device, with each one of its channels capturing the propagating wavefront almost simultaneously. When searching for similar LAWs within a time frame through all the channels of the catheter, repeating patterns corresponding to AF dynamics are easier to be found, leading to accurate and robust results, even in the complex cases of CFAEs. The method proposed highlights the repetitiveness of LAWs through neighboring channels without defying high amplitude activations that are not quite repeated, ensuring the efficient and wiser detection of LAWs.

\section{MATERIALS}

The database consisted of 34 multichannel recordings of bipolar AF EGMs, undergoing CA. Data was obtained using a LabSystem ${ }^{\circledR}$ Pro recording system (Boston Scientific, Marlborough, MA, USA) with sampling frequency of $1 \mathrm{kHz}$.

As catheter moves along the atrial tissue, it is quite common for one or more channels not to record cardiac activity at some time during the process. Such channels were not analyzed, while the recordings were chosen for analysis only if they contained 4 or more functional channels.

In total, 4 out of the 34 recordings did not meet the criteria and were excluded. Each one of the remaining 30 recordings that were analyzed consisted of 4 to 6 channels presenting high CFAEs activity, after the threshold criterion was applied. Signal length of each recording varied from 5 to $15 \mathrm{~s}$ and mean cycle length was $137 \pm 12.87 \mathrm{~ms}$.

Activations were firstly annotated manually by an experienced clinician, which did not participate in the algorithm development and therefore had no idea of the searching pattern that would be followed.

\section{METHODS}

\section{A. Data preprocessing}

Firstly, signals were filtered with a 3rd order Butterworth bandpass filter with cut-off frequencies set at 0.5 and 500 $\mathrm{Hz}$, so that the low and high frequency noises are reduced. Afterwards, a wavelet transform (WT) denoising technique, proved to efficiently denoise the signals without affecting the waveform [15] was applied.

Preprocessing continues with an altered step of the Botteron and Smith [16] preprocessing technique, with the lower cutoff frequency of the bandpass filter set at 20 instead of 40 $\mathrm{Hz}$. EGMs are then normalized from 0-1 values and an extra step for the enhancement of small amplitude activations is performed only in CFAEs and highly fractionated signals. Preprocessing steps are applied in the same way to all recording channels of all the EGMs.

\section{B. Data analysis}

EGMs are sorted according to their kurtosis value, which is computed in segments of 1 second and then is averaged over 5 seconds. Amplitude thresholds are set for every EGM, according to their kurtosis value as well, with lower amplitude thresholds assigned to more fractionated signals.

LAW detection is performed sequentially on the EGMs. Detection in the first EGM is performed based on the amplitude of the signal, followed by a cycle length (CL) based detection. The latter searches for LAWs in intervals longer than the median CL by decreasing the amplitude threshold proprortionally to the duration of the corresponding CL.

For each of the remaining channels, LAWs are primarily detected from the previous activations within time windows that vary according to their percentage of repetitiveness. Each previous activation has to be present in at least half of the channels analyzed so far, in order to be considered for the detection process on the remaining channels. CL-length based activation is performed in the rest of the channels, in the same way it is performed in the first channel that is analyzed and activation times are calculated by the barycenter of each LAW.

Once all EGMs are analyzed, a second-level control is performed through the activations of all EGMs, in order to delete any low amplitude activations that do not comply with the repetition threshold set. This step is introduced so that LAW detection in the first channel also passes through the detection filters that are applied in the rest of the channels and any bias regarding the different detection method of this channel is avoided.

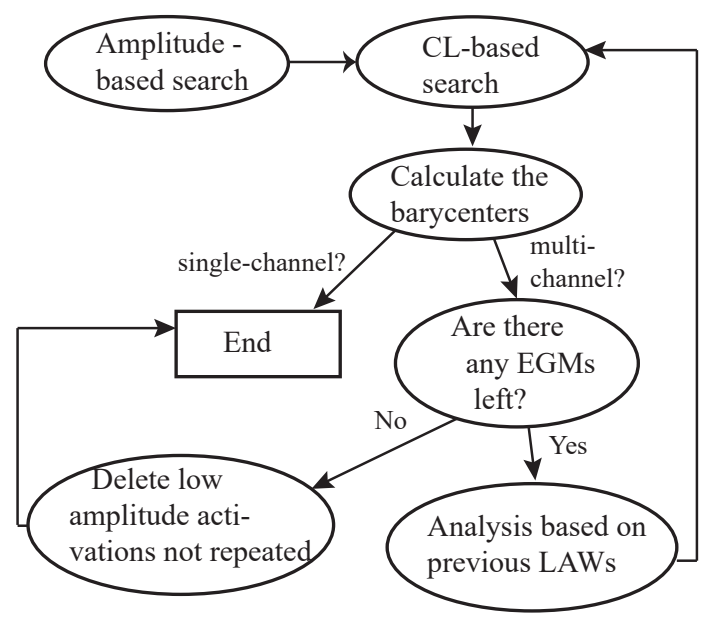

Fig. 1. Analysis steps of single channel and multichannel detection methods. Process is the same for the two algorithms in the beginning, but then multichannel method continues the analysis based on the previous LAWs detected. The final step deletes activations of low amplitude that are not repeated through the channels. 


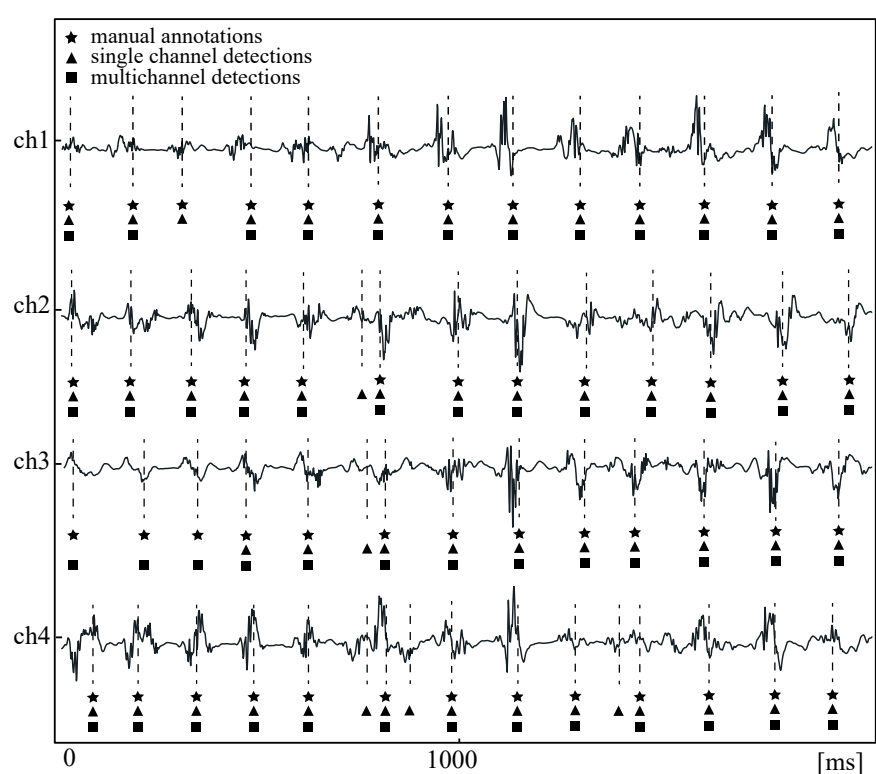

Fig. 2. Two seconds example of LAW detection performance on a 4-channe recording where manual annotations are marked with a star, single channel annotations are marked with a triangle and multichannel annotations are marked with a square.

\section{Evaluation}

For the evaluation of the algorithm, a single channel detector was created by simplifying the multichannel strategy up to the point that the first EGM was analyzed. This means that the detection was also based on a varying amplitude threshold and a second-line CL analysis. However, the activations of previous channels were not taken into consideration in this case. The differences in analysis steps between the two methods can be seen in Figure 1.

Preprocessing steps were the same in the two methods. Detected activations in both strategies were compared with manual annotations, noting a hit whenever they were closer than $40 \mathrm{~ms}$. Next, the corresponding confusion matrices were generated and accuracy, sensitivity and positive predictive value were obtained from them both in single and multichannel strategies. The Shapiro-Wilk and Levene tests were applied in both strategies to verify normality and homoscedasticity of false positive and false negative data from each channel in each recording. Finally, depending on the previous tests, Kruskal-Wallis and Mann-Whitney U-tests were applied to verify the statistical significance of the differences in single and multichannel strategies.

\section{RESUlts}

Figure 2 shows the differences between the two methods in a 2-second segment of 4 channels of one of the recordings. Multichannel analysis detected three LAWs in the beginning of channel 3 , that were ignored by sequential single channel analysis, while the latter detected several false positive activations in all the channels.

In channel 1, sequential single channel analysis correctly detects the first exclusive LAW, which was missed by mul-
TABLE I

ACCURACY, SENSITIVITY AND POSITIVE PREDICTIVE VALUE OF SEQUENTIAL SINGLE CHANNEL AND MULTICHANNEL METHODS.

\begin{tabular}{lcc}
\hline & Single channel & Mutlichannel \\
\hline Accuracy, \% & 89.65 & 90.82 \\
Sensitivity, \% & 96.47 & 93.89 \\
Positive Pred. Value (\%) & 92.70 & 96.52 \\
\hline
\end{tabular}

tichannel algorithm, but wrongly marks three more peaks as activations, while multichannel method correctly ignores them. In channel 2 , the only detection difference is limited to a LAW that was wrongly marked by sequential single channel analysis, as it is a shortcome form the neighboring activation.

Channel 3 shows three activations that only multichannel analysis managed to detect and a wrongly marked, single channel activation. The three exclusive sequential single channel detections of channel 4 are false positive and are correctly ignored by multichannel technique.

As can be seen from the table I, multichannel analysis performed better in terms of accuracy and positive predictive value. On the other hand, sensitivity rate was higher in sequential single channel method. False positive in this case is expressed as the number of peaks wrongly marked by the method as activations (oversensing) while the number of activations overlooked by the method is expressed in terms of false negative (undersensing). Oversensing was significantly higher in single channel analysis $(p<0.0001$, Kruskal-Wallis test), while undersensing was higher in the multichannel analysis, the difference was not statistically significant $(p=0.1676$, Mann-Whitney U-test).

\section{Discussion}

In spite of the fact that sensitivity was higher in the singlechannel case, prominent activations were more successfully marked by the multichannel technique. As results themselves can sometimes be misleading, oversensing and undersensing tendencies of single- and multichannel analysis should be a significant point of discussion in order to comprehend how each of the two approaches work. Considering that manual annotations, the reference point of the evaluation, is a human product, it is always susceptible to mishits, even when the experts who annotate the signals are highly experienced.

The significantly higher false positive rate of single-channel analysis suggests that the algorithm is overly sensitive to any peak that is beyond the determined amplitude threshold. On the other hand, higher undersensing rates means that multichannel algorithm is extremely selective in the detection process. This means that prominent activations, that most likely came from AF activity were detected, while secondary activations were ignored, as they are possibly expressing passive phenomena.

Most algorithms base their analysis on signal's properties criteria. In contrast, the presented technique simultaneously takes into considerations the AF spreading mechanisms. In the introduction it was hypothesized that reentries-wise analysis would bring more accurate results, as this technique is directly 
connected to an essential principle in the field of AF, the dominant reentries [17]. Developing an algorithm focused on detecting repeating patterns was a reasonable strategy and the hypothesis was confirmed.

Efficiency of the algorithm is based on but not limited to its structure, the careful design and the adaptive process through which EGMs are examined. Classification of EGMs according to their level of fractionation and initiation of the detection process with the least fragmented signal is a very wise choice, as the searching loop starts with the least bias possible. As a relatively low fractionated signal has more prominent, easy to find activations, chances are that LAW detection will have less false positive or missed detections.

Still it could be assumed that the activations-blind search that the least fragmented EGM is under could insert some sort of doubt on the functionality. However, considering that the final step consists of a second round control, deleting low amplitude activations that are not present in a specific percentage of the catheter's channels, the first EGM of the analysis is finally treated the same as the rest of the channels.

Signal preprocessing is often overlooked, but in fact it is the most important step of an analysis, as any modification of the signal affects the results obtained. WT filtering has been proved to keep the waveform intact, while noise is effectively removed [15]. Reduced cut-off frequency of $20 \mathrm{~Hz}$ in band-pass filtering of Botteron's and Smith's technique [16] enhanced the low frequency activations while the use of the hyperbolic tangent function in EGMs of high fractionation enhanced the low amplitude activations, facilitating the detection in the most complex cases.

The combination of the above preprocessing steps and the reentries-wise searching method contributed to a quite robust process due to which LAWs were correctly identified in most of the cases.

\section{CONCLUSIONS}

Analysis of EGMs is more efficient, when LAWs of neighboring channels is the principal searching criterion. A multichannel analysis can catch repeating phenomena such as stable or meandering rotors, that can be missed from traditional, single channel analysis. The efficiency and adaptability of the method suggest its application as a powerful tool in ablation and AF mapping procedures.

\section{ACKNOWLEDGMENTS}

Research supported by grants DPI2017-83952-C3 from MINECO/AEI/FEDER UE, SBPLY/17/180501/000411 from JCCLM and AICO/2019/036 from GVA.

\section{REFERENCES}

[1] P. Kirchhof, S. Benussi, D. Kotecha, A. Ahlsson, D. Atar, B. Casadei, M. Castella, and H.-C. Diener, "2016 ESC guidelines for the management of atrial fibrillation developed in collaboration with EACTS,' European Heart Journal, vol. 37, no. 38, 2016.
[2] E. J. Kim, X. Yin, J. D. Fontes, J. W. Magnani, S. A. Lubitz, D. D. McManus, S. Seshadri, R. S. Vasan, P. T. Ellinor, M. G. Larson, E. J. Benjamin, and M. Rienstra, "Atrial fibrillation without comorbidities: Prevalence, incidence and prognosis (from the Framingham Heart Study)," American Heart Journal, vol. 177, pp. 138-144, 2016.

[3] J.-p. Bassand, S. Virdone, S. Z. Goldhaber, S. Goto, S. Haas, W. Hacke, and G. Kayani, "Early Risks of Death, Stroke / Systemic Embolism and Major Bleeding in Patients with Newly Diagnosed Atrial Fibrillation : Results from the GARFIELD-AF Registry," Circulation, vol. 139, no. 6, pp. 787-798, 2019.

[4] F. Censi, C. Cianfrocca, and I. Purificato, "Atrial fibrillation and the $4 \mathrm{p}$ medicine," Annali dell'Istituto Superiore di Sanita, vol. 49, no. 3, pp. 247-248, 2013.

[5] E. P. Gerstenfeld and S. Duggirala, "Atrial fibrillation ablation: Indications, emerging techniques, and follow-up," Progress in Cardiovascular Diseases, vol. 58, no. 2, pp. 202-212, 2015.

[6] M. Haïssaguerre, P. Jaïs, D. C. Shah, A. Takahashi, M. Hocini, G. Quiniou, S. Garrigue, and A. Le Mouroux, "Spontaneous Initiation of Atrial Fibrillation by Ectopic Beats Originating in the Pulmonary Veins," The New England Journal of Medicine, vol. 339, no. 10, pp. 659-666, 1998.

[7] K. Yoshida, M. Ulfarsson, H. Tada, A. Chugh, E. Good, M. Kuhne, T. Crawford, J. F. Sarrazin, and N. Chalfoun, "Complex electrograms within the coronary sinus: Time- and frequency-domain characteristics, effects of antral pulmonary vein isolation, and relationship to clinical outcome in patients with paroxysmal and persistent atrial fibrillation," Journal of Cardiovascular Electrophysiology, vol. 19, no. 10, pp. 10171023, 2008.

[8] H. V. Bhatt and G. W. Fischer, "Atrial Fibrillation: Pathophysiology and Therapeutic Options," Journal of Cardiothoracic and Vascular Anesthesia, vol. 29, no. 5, 2015.

[9] H. Theres, R. W. Stadler, L. Stylos, M. Glos, T. Leuthold, G. Baumann, S. D. Nelson, and M. W. Krucoff, "Comparison of electrocardiogram and intrathoracic electrogram signals for detection of ischemic st segment changes during normal sinus and ventricular paced rhythms," Journal of Cardiovascular Electrophysiology, vol. 13, no. 10, pp. 990-995, 2002.

[10] K. T. Konings, C. J. Kirchhof, J. R. Smeets, H. J. Wellens, O. C. Penn, and M. A. Allessie, "High-density mapping of electrically induced atrial fibrillation in humans," Circulation, vol. 89, no. 4, pp. 1665-1680, 1994.

[11] B. Sunsaneewitayakul, M. Schwab, C. Khunnawat, T. Vasavakul, J. McKenzie, T. Ngarmukos, E. Kosar, and K. Nademanee, "A new approach for catheter ablation of atrial fibrillation: mapping of the electrophysiologic substrate," Journal of the American College of Cardiology, vol. 43, no. 11, pp. 2044-2053, 2004.

[12] L. Faes, G. Nollo, R. Antolini, F. Gaita, and F. Ravelli, "A method for quantifying atrial fibrillation organization based on wave-morphology similarity," IEEE Transactions on Biomedical Engineering, vol. 49, no. 12 I, pp. 1504-1513, 2002.

[13] R. Dalvi, A. Suszko, and V. S. Chauhan, "Graph search based detection of periodic activations in complex periodic signals: Application in atrial fibrillation electrograms," Canadian Conference on Electrical and Computer Engineering, vol. 2015-June, no. June, pp. 376-381, 2015.

[14] J. Ng, V. Sehgal, J. K. Ng, D. Gordon, and J. J. Goldberger, "Iterative method to detect atrial activations and measure cycle length from electrograms during atrial fibrillation," IEEE Transactions on Biomedical Engineering, vol. 61, no. 2, pp. 273-278, 2014.

[15] M. Martínez-Iniesta, J. Ródenas, R. Alcaraz, and J. J. Rieta, "Waveform integrity in atrial fibrillation: The forgotten issue of cardiac electrophysiology," Annals of Biomedical Engineering, pp. 1-18, 2017.

[16] G. W. Botteron and J. M. Smith, "A technique for measurement of the extent of spatial organization of atrial activation during atrial fibrillation in the intact human heart," IEEE Transactions on Biomedical Engineering, vol. 42, no. 6, pp. 579-586, 1995.

[17] F. Atienza and J. Jalife, "Reentry and atrial fibrillation," Heart Rhythm, vol. 4, no. 3, pp. S15-S16, 2007. 